Roger Chartier, La Main de l'auteur et l'esprit de l'imprimeur, Paris, Gallimard, coll. « Folio histoire », 2015, 406 p.

\title{
Stéphane Pétermann
}

\section{(2) OpenEdition}

Journals

Édition électronique

URL : https://journals.openedition.org/genesis/1613

DOI : 10.4000/genesis. 1613

ISSN : 2268-1590

Éditeur :

Presses universitaires de Paris Sorbonne (PUPS), Société internationale de génétique artistique littéraire et scientifique (SIGALES)

\section{Édition imprimée}

Date de publication : 9 mai 2017

Pagination : 223-224

ISBN : 979-1023-105636

ISSN : 1167-5101

\section{Référence électronique}

Stéphane Pétermann, «Roger Chartier, La Main de l'auteur et l'esprit de l'imprimeur, Paris, Gallimard, coll. « Folio histoire », 2015, 406 p. », Genesis [En ligne], 44 | 2017, mis en ligne le 05 juin 2017, consulté le 18 mars 2023. URL : http://journals.openedition.org/genesis/1613; DOl : https://doi.org/10.4000/ genesis. 1613 
et la quasi-impossibilité pour le lecteur de suivre les cheminements proposés. Ce constat débouche sur une conclusion à la fois encourageante et paradoxale. Ries a produit rien moins qu'un véritable hypertexte de papier, comme le montre la présence de pointeurs et de liens affectés d'un contenu sémantique. Dès les années quatre-vingt-dix, la critique génétique française a souligné l'impasse dans laquelle était enfermée l'édition génétique si elle devait être réalisée dans le cadre traditionnel d'un livre imprimé sur du papier, et elle a montré que l'hypertexte apporte des solutions satisfaisantes si l'on veut surmonter ces impossibilités 5 . Or Ries lui-même fait référence à la TEI et à son module génétique, qui représentent un point d'aboutissement technologique et théorique des réflexions menées dans les années quatre-vingt-dix. Mais il est plus que discret sur les raisons qui l'ont amené à choisir le cadre traditionnel de l'édition papier pour présenter son travail éditorial : outre la référence à la TEI, mais à propos des opérations d'écriture, la seule mention du problème est enfouie dans une note de la page 107, où Ries écrit : «L'idéal serait [...] une édition numérique qui mette de manière exhaustive les carnets à la disposition du lecteur et lui permette en même temps d'emprunter les chemins de lecture paradigmatique à l'aide d'une graphical user interface dynamique». À s'user les yeux sur les codages qu'il a élaborés, on se demande vraiment pourquoi il n'a pas opté pour cette édition numérique «idéale », alors même on le sait par ailleurs - qu'il ne souffre pas d'allergie informatique, puisqu'il est un des pionniers de l'exploration génétique du contenu des disques durs...

À cette - importante - réserve près, il faut saluer l'ouvrage de Ries pour la qualité et la précision de son travail éditorial. Souhaitons que le présent compte rendu donne à quelques généticiens francophones le désir d'en prendre connaissance et d'y puiser des matériaux de première qualité pour nourrir la discussion théorique et pratique autour de la réalisation d'éditions génétiques.
Roger Chartier, La Main de l'auteur et l'esprit de l'imprimeur, Paris, Gallimard, coll. «Folio histoire», 2015, 406 p.

\section{Compte rendu par Stéphane Pétermann*}

Roger Chartier réunit dans cet ouvrage des études parues entre 2001 et 2014, dans des versions retravaillées, auxquelles il joint un essai inédit intitulé «Textes sans frontières », formant le chapitre IV. En dix chapitres ainsi nouvellement articulés, Chartier dresse un panorama des œuvres littéraires

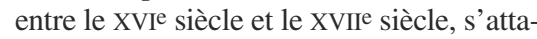
chant à «les replacer dans leur historicité propre» (p. 12), à en restituer les modes de production, de circulation et de lecture, à comprendre les conditions de leur réalisation. Pour ce faire, l'historien s'appuie sur la matérialité des œuvres, ou plutôt sur leurs matérialités, puisque aussi bien il distingue celle du livre, celle du texte et celle de la page, qui toutes entrent dans le processus complexe de fabrication et de publication de l'écrit. Pour Chartier, le texte lui-même n'est pas un objet immatériel, «si l'on entend par là les formes de son inscription manuscrite ou imprimée qui, tout en fixant l'œuvre, lui donnent mobilité et instabilité» (p. 14-15).

À travers l'étude de grandes œuvres telles que Don Quichotte ou Hamlet, Chartier rappelle quelques réalités trop souvent ignorées, même des spécialistes. La manière dont le lecteur du XXI ${ }^{\mathrm{e}}$ siècle perçoit et définit le fait littéraire s'est construite au cours de l'histoire, et doit beaucoup à la période étudiée ici, que bornent l'invention de l'imprimerie et l'avènement de l'auteur moderne - à cet égard, le titre retenu est particulièrement heureux, puisqu'il met en évidence les principales instances actives dans la création littéraire : les écrivains et leurs outils affrontant la page manuscrite, les imprimeurs, typographes et éditeurs donnant cadre et forme à leurs écrits.

Dans son avant-propos, l'auteur affirme sa volonté de «contribuer aux interrogations inspirées par les mutations contemporaines de la culture écrite» (p. 17). Non pas, ce qui serait hasardeux, en convoquant le passé pour prédire le futur, mais en précisant les notions faisant débat aujourd'hui, «qui ont fondé la définition de l'œuvre comme œuvre, la relation entre l'écriture et l'individualité et la propriété intellectuelle» (p. 18). Chartier ne prétend pas répondre à la question «Qu'est-ce que la littérature?», mais il concourt de manière déterminante à comprendre comment le fait culturel que nous appelons littérature s'est construit, entre manuscrit autographe et presse typographique. Ne croyant pas à la «mort de l'écrit», l'historien avance l'hypothèse que «la coexistence actuelle [...] entre différentes modalités de l'écrit, manuscrit, imprimé et électronique » est «sans doute durable» (p. 18).

La force de cet ouvrage «consacré aux objets et aux pratiques de la culture écrite» (p. 267), c'est de questionner, en en retraçant l'histoire - les délimitations - les définitions des notions qui leur sont liées. Chartier nuance le poncif des «pouvoirs» de l'imprimerie, et discute la question de l'autorité exercée sur l'écrit : dans le chapitre VIII, il montre par exemple qu'aux XVI et XVIII siècles, la ponctuation dépend moins des auteurs que des scribes, des compositeurs et surtout des correcteurs. Plus loin, il s'interroge simplement : «Qu'est-ce qu'un livre?» Faisant l'historique de la constitution des archives littéraires, il souligne leur lien étroit avec la «fétichisation de la main de l'auteur», et leurs effets sur les «contours de l'œuvre». Il y a, note-t-il, des auteurs «avec ou sans archives », et cette distinction n'est bien sûr pas indifférente à qui est réduit à «écouter les morts avec les yeux» (p. 9), pour reprendre la formule d'ouverture du livre. En outre, «les significations des œuvres changent même lorsque leur texte ne change pas» (p. 201), remarque Chartier, qui cite ailleurs Bourdieu (reprenant un propos de Joseph Levinson) allant dans le même sens : «On oublie qu'un livre change par le fait qu'il ne change pas alors que le monde change» (p. 296). Rien d'étonnant à ce que, parvenu au terme de son parcours, et commentant «Pierre Ménard,

(*) Université de Lausanne. 
auteur du Quichotte» de Jorge Luis Borges, Chartier achève sur des lignes qui évoquent «l'impossible rêve d'une œuvre toujours identique à elle-même» (p. 299). À l'illusion d'un âge d'or où l'écrit littéraire aurait connu un statut et une légitimité incontestables aujourd'hui mis à mal -, Chartier substitue la vision d'une ère moderne foncièrement complexe et dynamique.

Intitulée «Textes sans frontières» (chapitre IV), l'étude jusque-là inédite illustre bien ce parti qui attribue au texte un destin et des vies. Chartier y analyse en détail les traductions et éditions successives de la Brevísima relación de la destruyción de las Indias de Bartolomé de Las Casas (1552), ouvrage qui a joué un rôle de premier plan dans la propagation de la «légende noire» anti-espagnole. D'abord imprimé à Séville, le texte date de dix ans plus tôt, et une copie manuscrite en a été conservée. De retour d'Amérique, Las Casas dénonce dans ces lignes les atrocités de la colonisation telle qu'elle est organisée depuis les lois de Burgos de 1513. Dans un «Argument du présent sommaire» de l'édition de 1552, Las Casas précise que sa «relation» a été d'abord orale, et que la mise par écrit devait servir à alerter le roi Très Catholique de la situation dans ses colonies américaines. Chartier indique que le document a dû faire effet, puisque des réformes sont ordonnées à la fin de l'année 1542 déjà. Pourquoi alors le faire imprimer? Parce qu'en 1552, Las Casas est déçu de l'évolution des choses, et qu'il veut en appeler une nouvelle fois au roi, sous une forme «que Son Altesse [...] pût lire plus aisément» (p. 115). Pour donner plus de poids à son argumentaire, Las Casas fait imprimer en tout huit traités - dont la Brevísima relación - qui sont publiés sous une seule et même reliure. Chartier montre qu'ensuite, les trois traductions du texte publiées en terres protestantes (aux Pays-Bas espagnols et en Angleterre, entre 1578 et 1583) obéissent à des intentions de dénonciation politique, dans le contexte de la lutte des Pays-Bas contre leur souverain catholique, et dans celui de la rivalité anglo-espagnole de la fin du XVIe siècle. Autre usage et nouvelle interprétation, dans l'édition du texte parue à Francfort en 1598, dans le contexte des guerres religieuses : les cruautés espagnoles illustrées par Théodore de Bry servent à montrer ce dont les catholiques sont capables de commettre envers les peuples et les nations ayant adopté la Réforme. À Venise en 1626, à Barcelone en 1646 puis à Londres en 1656, de nouvelles éditions font écho aux préoccupations et aux intérêts politiques contemporains. Puis, à la fin du XVIIe siècle, le texte ressurgit dans plusieurs éditions en français, publiées à Paris, Amsterdam et Londres, qui présentent curieusement la Brevísima relación comme un «intéressant récit de voyage» (p. 136), à ranger aux côtés d'autres souvenirs pittoresques. Les descriptions des atrocités sont adoucies, quoique bien présentes, et des avertissements tentent d'en amoindrir la portée. Dernier avatar enfin, le texte paraît à nouveau en espagnol, à plusieurs reprises, dans les années 1810-1820 où les nations d'Amérique du Sud se soulèvent contre leurs maîtres européens.

Chartier clôt cet inventaire éclairant en remarquant: «La signification d'un texte ne dépend pas seulement de sa lettre. Elle est toujours construite par un double contexte : celui, historique, défini par les attentes de ses lecteurs, celui, matériel, donné par la présence dans le livre qui le publie de textes qui ne sont pas l'œuvre elle-même » (p. 143). En proposant d'associer histoire culturelle et critique textuelle, La Main de l'auteur et l'esprit de l'imprimeur offre d'innombrables pistes invitant à redécouvrir des œuvres de la tradition qui peuvent sembler figées dans leur interprétation.

Le Livre dit. Entretiens de «Duras filme », éd. Joëlle Pagès-Pindon, Paris, Gallimard, coll. «Les cahiers de la $\mathrm{NRF} », 2014,240 \mathrm{p}$.

\section{Compte rendu par Sandrine Vaudrey-Luigi*}

Tout commence avec la pièce Agatha, publiée le 3 mars 1981 aux Éditions de Minuit. Marguerite Duras entreprend aussitôt d'adapter son texte au cinéma avec une équipe relativement réduite. Dans le même temps, les proches qui l'entourent, Yann Lémée devenu très vite Yann Andréa - entré dans sa vie l'année précédente - son fils Jean Mascolo, surnommé Outa, et un ami, Jerôme Beaujour, la filment en train de réaliser son adaptation d'Agatha en vue de faire eux-mêmes un film intitulé Duras filme, documentaire qui paraît conjointement au film Agatha et les lectures illimitées, en octobre $1981 \ldots$ Le ton est donné : le volume édité par Joëlle Pagès-Pindon est le reflet d'une stratification et d'une mise en abyme jouant sur l'écrit, l'image, l'oral.

Le Livre dit. Entretiens de «Duras filme» est un volume provenant d'archives personnelles de Jean Mascolo, que ce dernier a confié à une des spécialistes de Marguerite Duras, Joëlle Pagès-Pindon. Ces archives consistaient en trois cassettes audiovisuelles contenant environ six heures d'enregistrement, plus précisément de rushes, c'est-à-dire de l'ensemble des prises de vue filmées en vue du montage de Duras filme: la première partie du volume, «Les entretiens de Duras filme», en propose une transcription. Mais à cette première strate s'en ajoute une autre puisque la seconde partie du volume est issue d'un dossier d'archives portant la mention «écrits à déchiffrer», composé de feuillets dactylographiés transcrivant des passages de Duras filme: il s'agissait pour J. Pagès-Pindon d'établir un texte à partir d'une transcription dactylographiée de Duras filme, transcription abondamment revue par Duras...

Précédant ces deux sections, la «Préface» et la «Note sur l'édition du texte» apportent plusieurs formes d'informations. La première partie de la préface contextualise les deux sections du volume en mettant en évidence l'importance du livre de chroniques L'Été 80 ainsi que celle de l'entrée dans la vie de l'écrivain de Yann Andréa - «vecteur de son imaginaire et [...] centre scriptural de sa création» (J. PagèsPindon, p. 17) - à la fin de l'été 1980. C'est également l'occasion de souligner à quel

(*) Université Sorbonne Nouvelle-Paris 3, Clesthia. 